\title{
26351 - EVALUATION OF MATERNAL AND NEONATAL EFFECTS OF ADDING TRAMADOL TO LIDOCAINE IN EPIDURAL ANESTHESIA FOR CESAREAN SECTION
}

\section{Farnad Imani Assistant Professor Of Anesthesiology, Valiollah Hassani, Zahra Taghipour, Faranak Rokhtabnak, Iran University Of Medical Sciences, Tehran, TEHRAN, Iran}

\section{INTRODUCTION:}

The goal of this study is comparison of adding different doses of tramadol to $2 \%$ lidocaine in epidural anesthesia for elective cesarean section.

\section{METHODS:}

After ethical approval, this study involved 90 pregnant patients, age between $20-40 \mathrm{yrs}$, with ASA physical status I or II candidated for elective cesarean section under epidural anesthesia. Epidural anesthesia was performed in lateral position by 18-gauge epidural needle, through L3-4 space. Epidural catheter for additional injections was indwelt. Patients were randomly allocated in three groups. Group L $(\mathrm{n}=30)$ received $20 \mathrm{ml} 2 \%$ lidocaine with epinephrine (1:200.000). In group LT50 $(n=30)$ and LT100 $(n=30)$ tramadol 50 and $100 \mathrm{mg}$ added to above epidural solution. For additional intraoperative analgesia, $2 \%$ lidocaine $5 \mathrm{ml}$ through epidural catheter, and intravenous sufentanil was administrated. Postoperative analgesia was provided by intramuscular meperidine. Onset of sensory block at T6, highest level of sensory block, two dermatome regression, onset and duration of complete motor block, sedation score, total intraoperative drug consumption (lidocaine and sufentanil), neonatal apgar score, duration of postoperative analgesia, time to first postoperative analgesic request, total $12 \mathrm{hrs}$ postoperative analgesics consumption (im meperidine), complications, and demographic data were recorded.

\section{RESULTS:}

There were no differences between the groups in demographic data, duration of anesthesia and surgery. Although, in LT100 group, onset of complete motor block and sensory block at T6 was less than two other groups, highest level of sensory block and two segment regression and duration of motor block between LT50 and LT100 groups were no differences, but were higher and more prolong than L group. Also, average intraoperative lidocaine and sufentanil consumption between LT50 and LT100 groups were no differences, but were less than L group. The incidence of maternal complications, neonatal apgar score was not statistically significant between three groups. In LT50 and LT100, time to first postoperative analgesics requirement was statistically prolonged and average meperidine consumption was less than L group.

\section{DISCUSSION:}

We found that addition of tramadol to $2 \%$ lidocaine offers an advantages over lidocaine alone for intraoperative anesthesia and postoperative analgesia in pregnant patients undergoing epidural anesthesia without increased maternal and neonatal complications. Keywords: tramadol, lidocaine, epidural anesthesia, cesarean section. 
REFERENCES:

Can J Anaesth 1998 Jul;45(7):631-4 